\title{
Varying Cardinality in Metonymic Extensions to Nouns
}

\author{
Helmut Horacek \\ Universität des Saarlandes \\ F.R. 6.2 Informatik \\ Postfach 151150 \\ D-66041 Saarbrücken, Germany \\ email: horacek@cs.uni-sb.de
}

\begin{abstract}
Meaning shifting phenomena such as metonymy have recently attracted increasing interest of researchers. Though these phenomena have been addressed by plenty of computational methods, the impacts of cardinalities of metonymically related items have been widely ignored in all of them. Motivated by this lack of analysis, we have developed a method for representing expectations and knowledge about the cardinalities of metonymically related entities and for exploiting this information to build logical forms expressing metonymic relations, the entities related, and their cardinalities. The representation of lexically motivated knowledge is realized as an enhancement to Pustejovsky's Generative Lexicon, and the process of building logical forms takes into account overwriting of default information and mismatch of cardinality requirements. Our method enables a precise attachment of sentence complements, and it supports reference resolution in the context of metonymic expressions.
\end{abstract}

\section{Introduction}

Meaning shifting phenomena such as metonymy have recently attracted increasing interest. Computational approaches to these phenomena aim at inferring implicitly represented relations, predicting meaning shifts of nouns or verbs, expressing restrictions on these meaning shifts in dependency of context- or language-specific factors, and facilitating reference resolution. Measures to achieve these issues include representation of default knowledge and various sorts of inference methods and constructive procedures. However, the entities in the texts examined almost always appear in singular form so that issues of cardinality of metonymically related items have been widely ignored by the approaches made so far.

Motivated by this lack of analysis, we have examined metonymic expressions by varying cardinalities of the items appearing explicitly or implicitly, to analyze effects of these alternations. The results have inspired us to build increasingly explicit versions of logical forms, and to formulate conditions on pronominal accessibility. The insights gained improve analysis methods for relating contextual specifications to the appropriate entity, and for supporting reference resolution to entities related implicitly.

This paper is organized as follows. We review computational approaches to metonymy. Then we illustrate the phenomena investigated. We elaborate suitable techniques to deal with these phenomena, that is, an enhancement to entries in the Generative Lexicon, and a procedure for building a logical form. Finally, we discuss impacts of our analysis on pronominal resolution.

\section{Approaches to Metonymy}

Metonymy is a natural language phenomenon that contributes to expressing information in an effective and economic way. It involves what has been termed 'transfers of meaning' by (Nunberg 1995), i.e., the meaning of some constituent does not correspond to what can be expected according to the syntactic and semantic environment the speaker is "using one entity to refer to another that is related to it" (Lakoff and Johnson 1980). For example, in the utterance "The ham sandwich is waiting for his check", it is not literally the ham sandwich, which wants to pay, but the person who ordered it.

Computational approaches such as the NL database interface TEAM (Grosz et al. 1988) are concerned with inferring implicitly expressed metonymic relations, mostly in English; some analyses consider German (Horacek 1996) and French (Kayser 1988, Pustejovsky and Bouillon 1995). Prominent representatives include Fass' program met* (1991), which makes use of formal definitions of several kinds of metonymic relations, Sowa's conceptual graphs (1992), in which an a priori unspecific relation is inserted between a concept of the type expected and the concept appearing on the surface, and the TACITUS system (Hobbs et al. 1993) which treats metonymy as a special case of reference resolution, in a uniform abduction process to "find the best explanation for the observables". Altogether, these approaches have two characteristic properties: (1) The conditions expressing when leaving a metonymic relation implicit or not is possible are too unconstrained to cover a larger number of examples in several languages, 
or to generate sentences with metonymic expressions systematically. (2) The intended and the literal referent always appear in singular definite form. There are only three approaches which in some aspects deviate from this characterization.

Pustejovsky's Generative Lexicon (1991) addresses the first aspect. He proposes a Theory of Qualia, with an explanation of systematic polysemy. Applying type coercion enables one to arrive at cases of ordinary metonymy which can be grounded in terms of the semantics of lexemes, as well as at word senses which Pustejovsky has termed logical metonymy, like the reading of a book in the sentence "Mary enjoyed the book". Such contexts reflect prototypical knowledge derived from AGENTIVE or TELIC roles of the lexical entry for 'book', which are prominent roles in the Qualia Structure of nouns. Particularities of the Qualia Structure of nouns regulate the acceptability of leaving a metonymic relation implicit (Pustejovsky and Bouillon 1995).

Stallard (1993) indirectly addresses the second aspect by taking into account scoping relations and impacts on pronominal reference. He introduces a distinction between referential and predicative metonymy, depending on whether the intended or the literal argument is accessible for subsequent pronominal reference. This distinction manifests itself in different scope relations that hold between these arguments in the corresponding logical forms. We will argue against his usage of scoping and the resulting strict distinction of pronominal accessibility.

Markert and Hahn (1997) address interactions of metonymic relation extension and anaphora resolution, which enables them to handle textual ellipsis references. They apply extensive language independent conceptual definitions with relational path classifications and preference rules. In their corpus, there are also cases of indefinite metonymic NPs, which is an indication for metonymic relations to several objects.

Though neither Pustejovsky's nor Stallard's approach address cardinalities, we show that both can be extended accordingly: we augment the Generative Lexicon by representing cardinality information, and techniques for building logical forms are enhanced to yield more precise specifications of the metonymically related entities.

\section{Phenomena Investigated}

For a number of metonymic relations, such as PRODUCER for PRODUCT ("I bought a Ford"), ARTIST for ARTWORK ("He plays Bach"), as well as eventualities involved in logical metonymy, cardinalities are not a problem because the literal referents are expressed as proper names. For other metonymic relations, especially ORGANIZATION for MEMBER and PART for
WHOLE, several complications may arise, as the following examples demonstrate. Let us start with two contrastive sentences (1) and (2), taken from (Lakoff and Johnson 1980), and (Hobbs et al. 1988), respectively (see also (Horacek 1994)):

(1) The ham sandwich is waiting for his check. (1a) He is getting impatient.

(1b) It is $2 \$$.

(2) The Boston office called.

? (2a) He was angry.

(2b)It is our head quarter.

(2c) They want us to organize a meeting.

Following Stallard, (1) is interpreted as an example of referential reading, while (2) as an example of predicative reading: (1) can be rephrased more explicitly by The man $_{x}$ who has eaten a ham sandwichy is waiting for his $s_{x}$ check, while (2) in a similar way gets expanded to The Boston office $_{x}$ represented by one of its $s_{x}$ employees call- $^{-}$ $e d$. These reformulations suggest that the man in (1) and the Boston office in (2) have wider scope in Stallards representation than the ham sandwich in (1) and the employee in (2), which predicts pronominal accessibility in (1a) and (2b), as opposed to (1b), (2a) and (2c). We challenge this analysis with evidence from the examples above. Pronominal reference in (1b) is also possible, but may be less common than in (1a). (2c) seems even more natural than (2b), only (2a) is unclear.

Further complications arise when variations of cardinality in sentence (1) (see sentences (3) to (6) and their follow-ups), and variation of circumstances in sentence (2) (see the follow-ups of sentences (7) and (8)) are considered. For dishes made of animals ('the mussels'), complications arise through interference between animals and persons as pronominal referents. Because we want to study the effects of cardinality variations per se, we avoid such examples.

(3) The pizzas are waiting for their checks.

? (3a) He/she is getting impatient.

(3b) They are getting impatient.

(4) The fruit dumplings is/are waiting for his/her/their check(s).

(4a) $\mathrm{He} /$ she/they is/are getting impatient.

(5) The meat plate is/are waiting for his/her/ their $\operatorname{check}(\mathrm{s})$.

(5a) $\mathrm{He} /$ she/they is/are getting impatient.

(6) Table 7 is/are waiting for his/her/their $\operatorname{check}(\mathrm{s})$.

(6a) He/she/they is/are getting impatient.

These sentences demonstrate that both intra- ((1) and (3)) and intersentential ((1a) and (3b)) prononminal reference work fine, if the literal referents (here, various sorts of food) and the real referents (here, the persons) agree in number. Otherwise, a variety of complications arise in in- 
trasentential reference, which also demonstrate a specificity of English. Whereas pronouns agree with the literal referent in most languages, it is the intended referent that determines verb agreement and pronominal reference in the same sentence in English. For example, metonymic extension to the expression 'fruit dumplings' is ambiguous in the sense that it can refer to one plate of dumplings to be eaten by a single person, or to several plates, each for another person (see the variants in (4)). Conversely, metonymic extension to the expression 'meat plate' can also be interpreted as a reference to several persons sharing that dish (see the variants in (5)). Finally, metonymic extension to the expression 'table' seems to be more neutral with respect to the number of persons sharing it (see the variants in (6)). Thus, the syntactic subject and the verb would not agree in number in English, when the default situation concerning these dishes is present. Hence, English is, in principle, more informative than other languages when the cardinality of the intended referent differs from the number of the literal referent. However, those expressions without subject/verb agreement are unlikely to occur in practice, since they appear to be strange.

Unlike with intrasentential reference, intersentential pronominal reference with number features deviating from the referent that is pronominally accessible intrasententially is possible due to default expectations about the cardinality of the real referents (compare complementary variants in (4a) and (5a)). It is more problematic in other cases (see (3a)). In the less precise reference by the table, all variants in (6a) are felicitous.

(7) The Boston office is represented in the meeting.

? (7a) He/she is an expert in marketing.

(7b) They are experts in marketing.

(7c) They always send someone to meetings.

(8) The Boston office will meet for an excursion today.

* (8a) He/she likes to walk.

(8b) They will make a lunch break at $2 \mathrm{pm}$.

(8c) They like to organize social events.

The following sentences ((2), (7), (8), and their follow-ups) involve slightly harder restrictions. Plural pronominal references as in sentences (7b), (7c), (8b) and (8c) are felicitous, but there is a difference between the sets of entities the plural pronouns refer to. While in $(7 \mathrm{c})$ and $(8 \mathrm{c})$, the pronouns refer to the entire set of employees of the Boston office, they more plausibly refer to the representatives in the meeting in (7b) and to the excursion participants in (8b). These examples indicate an additional demand on the treatment of cardinalities and referential accessibility of metonymic expressions: a distinction is to be made between the entities referred to metonymi- cally (here: employees of the Boston office), and those of its members involved in the event expressed by the sentence (here: the meeting and the excursion). For the restaurant scenario, these sets of persons are mostly identical except to those cases where one person out of a group of persons eating together and referred to metonymically is the one who intends to pay.

(9) Which airlines serve food from Boston to New York?

(9a) In the first class?

As a further aspect of metonymic expressions, the last two examples demonstrate chaining of metonymic relations and the relevance of each set of items involved for the associated analysis. In sentence (9), the airlines are the literal, and the persons the real referents. However, relating these two entities directly by an employment relation is problematic, since it is impossible to connect the locality information (from Boston to New York) and the first class restriction to either of them. Linking this information appropriately requires explicit elaboration of the relation between the airlines and their employees to include the implicitly referred flights.

The consideration exposed so far primarily hold for English. Apart from the partitive construction, which seems to be a specificity of English in comparison to many other languages, the results can widely be transferred to other languages. However, there are a lot a language-specific subtleties which may influence the felicity or non-felicity of some of the expressions discussed in one particular language. In order to find out, to what extent other languages behave similar to English, we have asked native speakers of German, French, Italian, Spanish, Russian, and Vietnamese about the transferabillity of the English sentences to these languages. Though the results are subjective to a certain extent (only one speaker was available for most of these languages), some tendencies became apparent. Even sentence (1) was considered unacceptable in some languages, in which there is more emphasis on referring to persons explicitly. In Spanish, this seems to be caused syntactically, by the absence of personal pronouns, while the reasons seem to be more pragmatically or culturally related in French and Vietnamese, respectively. Moreover, references to objects ((1b) and (2b)) appeared unusal in some languages, including Vietnamese and Italian. Also in German, a demonstrative pronoun seems to be preferable to a personal pronoun. Finally, (2) is quite weird in Spanish, since the alternative 'From the Boston office _ called' exists (unlike in (7) and (8)). In constrast, precisely (2) is acceptable in Vietnamese, because only 'calling' is considered technical. 


\begin{tabular}{|c|c|c|c|c|c|}
\hline \multicolumn{2}{|l|}{ food $(x)$} & \multicolumn{2}{|c|}{$\operatorname{sandwich}(x)$} & \multicolumn{2}{|l|}{$\operatorname{pizza}(\mathbf{x})$} \\
\hline CONST & $=\{$ ingredients,$\ldots\}$ & CONST & $=\{$ ham, bread, ... $\}$ & CONST & $=\{$ dough, tomato, $\ldots\}$ \\
\hline FORMAL & $=\operatorname{eatable}(x)$ & FORMAL & $=\operatorname{eatable}(x)$ & FORMAL & $=\operatorname{eatable}(x)$ \\
\hline TELIC & $=\operatorname{eat}\left(\mathbf{e}^{\mathrm{T}}, \mathbf{y}, \mathbf{x}\right)$ & TELIC & $=\operatorname{eat}\left(\mathbf{e}^{\mathbf{T}}, \mathbf{y}, \mathbf{x}\right)$ & TELIC & $=\operatorname{eat}\left(\mathbf{e}^{\mathbf{T}}, \mathbf{y}, \mathbf{x}\right)$ \\
\hline AGENTIVE & $=\operatorname{cook}\left(\mathrm{e}^{\mathrm{T} T}, \mathbf{z}, \mathbf{x}\right)$ & AGENTIVE & $=\operatorname{prepare}\left(\mathbf{e}^{\prime T}, \mathbf{z}, \mathbf{x}\right)$ & AGENTIVE & $=\operatorname{bake}\left(\mathbf{e}^{\mathrm{T} T}, \mathrm{z}, \mathrm{x}\right)$ \\
\hline
\end{tabular}

Fig. 1. Some 'standard' examples of Qualia Structures, for 'food', 'sandwich', and 'pizza'

\section{Expressing Lexical Knowledge}

In order to capture distinctions between the varying interpretations of metonymic expressions, knowledge about the lexical items involved plays a crucial role. For adequately expressing this knowledge, we make use of entries in the Generative Lexicon (see Figure 1). Since the information represented there is insufficient for reasoning about cardinalities, we extend the entries in the Generative Lexicon, prominently the TELIC role, by quantifier specifications. In the original form, the entities involved (typically, the lexical item itself and some related entity) are implicitly quantified, and a typed event variable is used (an event may be a state (S), a process $(\mathrm{P})$, or a transition (T)). A similar exploitation of taxonomic knowledge in terms of cardinality restrictions has been undertaken for scope disambiguation in (Fliegner, 1988).

In the extended form, we introduce explicit quantifiers and scoping, and we optionally add sort restrictors to variables referred to by event predicates. We introduce new quantifiers to cover the cases elaborated in the previous section, in addition to the usual NL quantifiers EXIST and WH: SINGLE and MULTIPLE for a single resp. multiple objects without defaults, DEFSINGLE and DEFMULTIPLE for the same with defaults.

Figures 2 and 3 show entries in the Generative Lexicon with extended TELIC roles. The same extensions also apply to the AGENTIVE roles, but we do not elaborate this aspect here. Figure 2, for example, shows some sorts of food associated with different expectations about how many persons typically eat them. Fruit dumplings appear as sets (quantified by DEFMULTIPLE), to be eaten as a dish by a single person (quantified by DEFSINGLE). For the meat plate, cardinality relations are inverted. For a table in a restaurant, relations to food eaten at that table are not specified.

Unlike in the restaurant scenario, cardinality relations are less vague for some relations in organizations. Each office and each airline are supposed to employ several persons, and each person is working for one organization only, at least in his/her individual activities (this is expressed by the quantifiers SINGLE and MULTIPLE in the lexical entries in Figure 3). Each flight carries some set of people, each of which participates in one flight only (at the same time).

These extensions allow us to derive cardinalities for the referents involved in a metonymic expression - compare the entries for FRUITDUMPLING and MEAT-PLATE, as contrasting examples. To achieve this goal, the knowledge represented in the lexicon entries is used for building logical forms in which metonymic relations are made entirely explicit. The event predicates in the TELIC (or AGENTIVE) roles are exploited to infer the relation involved. Moreover, the new quantification specification yields crucial information to build an explicit logical form with cardinality specifications from concise surface expressions in a precise manner.

\section{Building Logical Forms}

Based on entries in the Generative Lexicon and on the context given by a sentence to be interpreted, logical forms can be built that represent the semantic relations involved more explicitly than this is the case with previous approaches. In a nutshell, metonymic extensions are tried according to specifications found in the lexicon, as long as the sort of an NP and the sort of the referring case role are incompatible. In addition, agreement between syntactic number and seman-

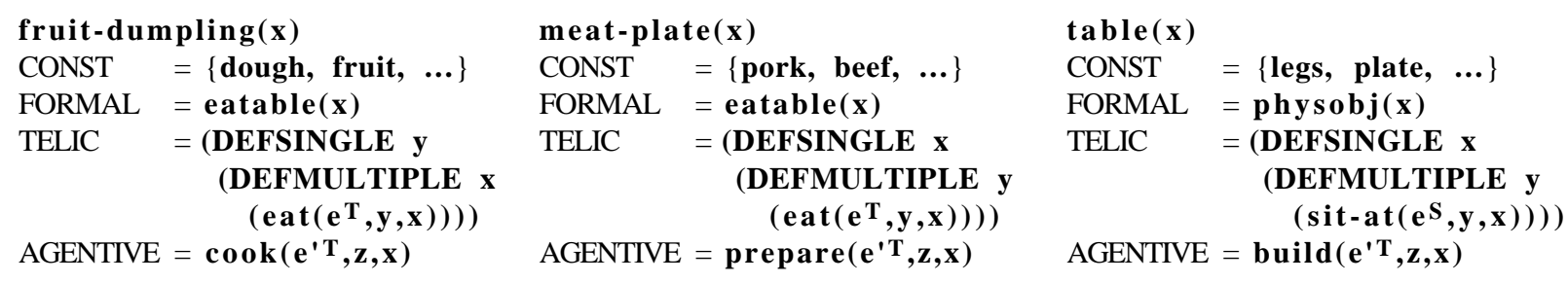

Fig. 2. Some 'extended' examples of Qualia Structures, for special food sorts and 'table' 


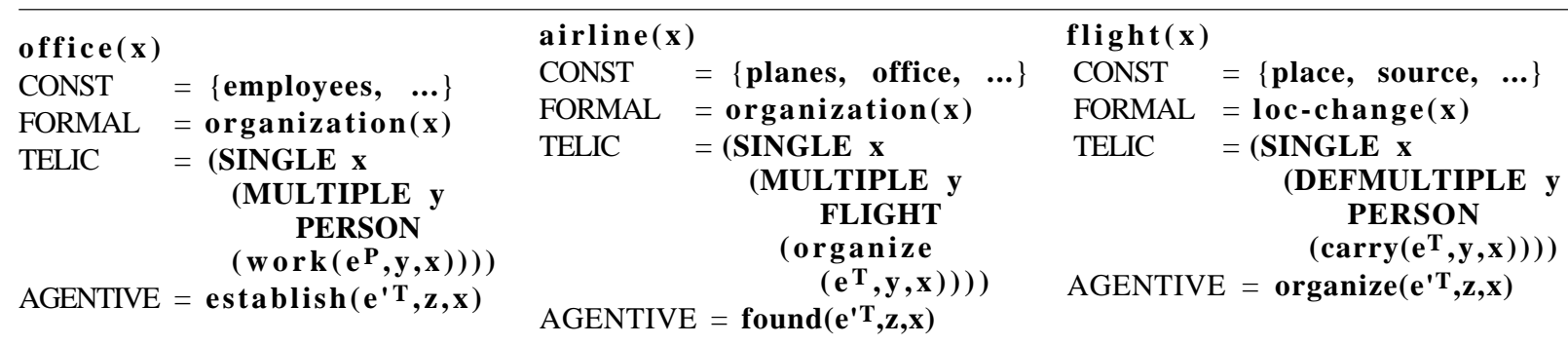

Fig. 3. Some 'extended' examples of Qualia Structures, for 'office', 'airline', and 'flight'

tic cardinality specifications is achieved, which may require overwriting defaults or introducing a new set of entities as a subset of a known set. In concrete, logical forms are built by pursuing the procedure in Figure 4. Logical forms appear as ( $\mathrm{Q} \times \mathrm{S}\langle\mathrm{P}\rangle$ ), where $\mathrm{Q}$ is a quantifier, $\mathrm{x}$ and $\mathrm{S}$ its associated variable and sortal restrictor, and $\langle\mathrm{P}\rangle$ the predication related. In step $2 \mathrm{a}$, metonymic extensions are carried out, which can potentially be chained, and in step $2 \mathrm{c}$ a final extension is performed in case of a cardinality mismatch. In the following, we illustrate the procedure by some examples. For sentence (4), "The fruit dumplings wants to pay", the initial logical form

(MULTIPLE x FRUIT-DUMPLING (WANT-PAY x)) contains a sortal incompatibility. Using the lexical entry for 'fruit dumplings' and expanding the expression according to the TELIC role yields
(SINGLE y PERSON

(MULTIPLE x FRUIT-DUMPLING

(AND (EAT y x) (WANT-PAY y))))

where the sortal incompatibility is removed. Note, that the cardinality of PERSON is singular, due to the inflection of the predicate 'wants'. In German, the quantifier is unspecific concerning the cardinality, because the sentence predicate would not give the same indication as this is the case in English. For another predicate, such an ambiguity may not be present, as in the example

$$
\text { (SINGLE x OFFICE (AND (BOSTONIAN } \mathrm{x} \text { ) }
$$$$
\text { (CALL } \mathrm{x})) \text { ) }
$$

Making use of the TELIC role in the lexical entry of 'office', as exposed in Figure 3, yields

(SINGLE x OFFICE (AND (BOSTONIAN x)

(MULTIPLE y PERSON (AND (WORK y x) $($ CALL y)))))

1. Build an initial logical form out of the surface expression.

The representation is composed as an expression of the form $\left.\left(\mathrm{Q}_{E} \mathrm{x}_{\mathrm{E}} \mathrm{S}_{\mathrm{E}}<\mathrm{P}\right\rangle\right)$ :

$\mathrm{x}_{\mathrm{E}}$ being the variable whose representation is to be extended (initially $\mathrm{x}$, the literal referent),

$\mathrm{QE}_{\mathrm{E}}$ being its quantifier, and $\mathrm{S}_{\mathrm{E}}$ its sort (initially $\mathrm{Q}$ and $\mathrm{S}$, associated with the literal referent), and $\langle\mathrm{P}\rangle$ being a structured representation of the sentence predicate and its modifiers.

$\mathrm{S}_{\mathrm{R}}$ is the sort required in the referring case frame, and $\mathrm{Q}_{\mathrm{R}}$ the quantifier of its case slot restrictions.

2. Extend the meaning of noun phrases which are involved in a sortal incompatibility.

2a. Build a metonymically extended expression by consulting lexical knowledge.

Merge the partial expression $\left(\mathrm{Q}_{\mathrm{E}} \mathrm{X}_{\mathrm{E}} \mathrm{S}_{\mathrm{E}}\langle\mathrm{P}\rangle\right)$ with the extended lexicon representation for $\mathrm{S}_{\mathrm{E}}$ :

$\left(\mathrm{Q}_{1} \mathrm{x}_{1} \mathrm{~S}_{1}\left(\mathrm{AND}<\mathrm{P}_{1}>\left(\mathrm{Q}_{2} \mathrm{x}_{2} \mathrm{~S}_{2}<\mathrm{P}_{2}>\right)\right)\right)-$

from the lexicon, $\left\langle\mathrm{Q}_{1}, \mathrm{x}_{1}, \mathrm{~S}_{1}\right\rangle=\left\langle\mathrm{QE}_{\mathrm{E}}, \mathrm{xE}_{\mathrm{E}}, \mathrm{S}_{\mathrm{E}}\right\rangle$ and $\left\langle\mathrm{Q}_{2}, \mathrm{x}_{2}, \mathrm{~S}_{2}\right\rangle=\left\langle\mathrm{Q}_{\mathrm{N}}, \mathrm{x}_{\mathrm{N}}, \mathrm{S}_{\mathrm{N}}\right\rangle$

if the referent with the same sort as $x_{E}$ has wider scope in the lexicon, or with inverted equalities.

$\langle\mathrm{P}\rangle$ is partitioned according to sortal compatibility of its components:

if $\mathrm{x}_{1}=\mathrm{x}_{\mathrm{E}}$ then $\left\langle\mathrm{P}_{1}\right\rangle$ contains parts that refer to $\mathrm{x}_{\mathrm{E}}$, sortally compatible with $\mathrm{S}_{\mathrm{E}}$, otherwise $\left\langle\mathrm{P}_{2}\right\rangle$.

The remaining parts of $\langle\mathrm{P}\rangle$ become $\left\langle\mathrm{P}_{2}\right\rangle$, if $\mathrm{x}_{1}=\mathrm{x}_{\mathrm{E}}$, and $\left\langle\mathrm{P}_{1}\right\rangle$ otherwise.

$2 \mathrm{~b}$.Test the compatibility of the newly inserted sort with the restrictions to be met.

If $S_{N}\left(S_{N}=S_{2}\right.$, if $S_{1}=S_{E}$, and $S_{N}=S_{1}$ otherwise $)$ is incompatible with $S_{R}$,

then repeat step $2 \mathrm{a}$ with $\mathrm{x}_{\mathrm{N}}, \mathrm{S}_{\mathrm{N}}, \mathrm{Q}_{\mathrm{N}}$ and $\left\langle\mathrm{P}_{\mathrm{N}}\right\rangle$ as $\mathrm{xE}_{\mathrm{E}}, \mathrm{S}_{\mathrm{E}}, \mathrm{Q}_{\mathrm{E}}$ and, $\langle\mathrm{P}\rangle$, respectively;

else $\mathrm{Q}_{\mathrm{R}}$ overwrites $\mathrm{Q}_{\mathrm{N}}$ if $\mathrm{Q}_{\mathrm{N}}$ is a default quantifier compatible with $\mathrm{Q}_{\mathrm{R}}$.

2c. Test the cardinality compatibility of the new sort with the restrictions to be met.

If $S_{N}$ is compatible with $S_{R}$, but $Q_{N}$ is incompatible with $Q_{R}$, insert MEMBER between $x_{E}$ and $x_{N}$.

If not the whole set of entities bound to xE participates in the eventuality, insert SUBSET instead.

Fig. 4. The procedure for building logical forms with extended metonymic relations 
which still contains a cardinality incompatibility. Further expanding this form by performing step $2 \mathrm{c}$ in the procedure leads to the insertion of a MEMBER relation, yielding

$$
\begin{aligned}
& \text { (SINGLE x OFFICE (AND (BOSTONIAN x) } \\
& \text { (MULTIPLE y PERSON (AND (WORK y x) } \\
& \text { (SINGLE z PERSON (AND (MEMBER z y) } \\
& \text { (CALL z)))))) }
\end{aligned}
$$

in which all incompatibilities are resolved. Proceeding in the same manner, the analysis of the sentence "The Boston office makes an excursion" yields a similar result, with only two minor deviations, partially grounded in the semantic difference between 'calling' and 'excursion making': (1) The variable $\mathrm{z}$ is quantified by MULTIPLE instead of SINGLE, and (2) the expression (SUBSET z y) replaces (MEMBER z y). However, obtaining precisely this representation, that is, performing the insertion of the SUBSET relation, additionally requires some sort of pragmatic knowledge: typically not all members of an organization participate in events such as excursions. Nevertheless, suitable ways to represent such domain-dependent pieces of knowledge adequately are delicate.

Finally, sentence (9), "Which airlines serve food from New York to Boston?", shows how chained metonymic extensions are handled:

(WH x AIRLINE (AND (SERVE x FOOD)

(SOURCE x NEW YORK) (GOAL x BOSTON)))

The first metonymic extension, based on the lexicon entry for 'airline' (see Figure 3), tentatively inserts 'flights' linked to 'airline' via an ORGANIZE relation, and yields

\section{(WH x AIRLINE (MULTIPLE y FLIGHT}

(AND (ORGANIZE x y) (SERVE y FOOD)

(SOURCE y NEW YORK) (GOAL y BOSTON))))

and the final operation based on the lexicon entry for 'flight' (see Figure 3) leads to a similar extension, inserting 'person' related to 'flight' via a CARRY relation:

(WH x AIRLINE

(MULTIPLE y FLIGHT (AND (ORGANIZE x y) (SOURCE y NEW YORK) (GOAL y BOSTON) (MULTIPLE z PERSON

(AND (CARRY y z) (SERVE z FOOD))))))

Note the distinguished treatment of the predications containing the variable which represents the phrase to be extended, as opposed to the previous examples. In all cases discussed so far, appearances of this variable are replaced by the new variable introduced in the course of an extension. Here, replacing $y$ by $z$ in the second extension step is only carried out in (SERVE y FOOD), while y remains unchanged in (SOURCE y NEW YORK) and (GOAL y BOSTON). This is because SOURCE and GOAL can be established as properties of flights, while CARRY needs a further extension to 'person' to be connected appropriately. Building explicit logical forms in this way demonstrates a number of achievements over other methods:

- Scoping of variables reflects their dependencies in the event they are involved in.

- More referents than just the real and the literal referent may be introduced, through chained metonymic extensions or through membership/subset insertions.

- An additional referent may provide a proper place to relate sentence complements.

Note, that there is a scoping difference between "one and the same person eating several fruit dumplings" and "several persons sharing a meat plate", which contrasts Stallard's approach.

Finally, we have to admit that this procedure is overgenerating, as it does not take into account the restrictions imposed on the use of metonymic expressions discussed in Section 3. The procedure is cooperative in the sense that it attempts to interpret a given metonymic expression, but it is not strong enough to distinguish felicity or infelicity of a metonymic expression, which may be due to various lexical and pragmatic factors.

\section{Impacts on Reference Resolution}

Empirically supported by the considerable number of examples discussed in section 3 , our approach is able to explain more pronominal references to metonymic expressions than others:

- Reference to literal and intended referents is possible in an increasing number of cases.

- Pronominal reference in plural form may have as antecedents distinguished sets of entities.

- Cross-language differences in the treatment of intersentential pronominal reference exist.

In order to express scoping relations among sets properly, the logical forms representing metonymic expressions with entities of cardinality greater than one must deviate from Stallard's methods. According to Stallard, pronominal reference to literal and real referents is regulated by their scope, which distinguishes referential from predicative kinds of metonymy. Unfortunately, this realization of metonymic extension is incompatible with the common use of scoping. However, we believe that Stallards distinction is in some sense artificial, because the felicity of pronominal reference seems to be more complex and influenced by other factors than scoping. For example, the sentence "the ham sandwich is waiting for his check" can be followed by some information useful to a novice waiter: "It costs $2 \$$." Moreover, the message "The Boston office called" can be followed by the remark "He spoke angrily" in some plausible contexts. Hence, it 
does not seem to be referential inaccessibility which makes many similar examples sound odd, but the rare occurrence and the low coherence in neutral contexts. For example, it is usually of minor interest whether the person calling on behalf of the Boston office is angry himself; it is the attitude of the responsible representatives at the office that is usually more interesting.

Given these pieces of evidence, reference resolution is supported by the explicit logical form built through our techniques, and it is additionally guided as follows:

\section{Intrasentential reference}

Possessive pronouns always relate to the intended referent. Since possessive pronouns in the same sentence agree with the real referent in English, while they agree with the literal referent in most other languages, only English sentences contain information about cardinality and gender of the intended referent. For example, the sentence 'the fruit dumplings is waiting for his check' carries the additional implication that there is one male person who wants to pay.

\section{Intersentential reference}

Reference through personal pronouns is possible to the literal and to the real referent, and to referents of the same sort but with possibly different cardinality as the real referent. Thus, all entities involved in a metonymic expression in its appearance in the explicit logical form are potential antecedents, except to internal elements of a metonymic chain. For example, following the sentence "The Boston office called", pronominal reference is possible to the office (the literal referent), to the caller (the real referent), and to the people at the office (differing from the caller by number only). However, 'the flights' appearing in the logical form representing the sentence "Which airlines serve diet food from New York to Boston?" are not pronominally accessible.

\section{Conclusion}

In this paper, we have presented an approach to deal with cardinality aspects of metonymic extensions to nouns. We have discussed a variety of constellations with pronominal references to implicitly related items, sometimes associated with subtle conditions, focusing on English, also including some language specificities. In order to build explicit logical forms with cardinality specifications, we have extended entries in Pustejovsky's Generative Lexicon by default quantifier specifications. Through exploiting these entries, metonymic extensions are introduced on the basis of events represented in the roles of the Qualia structure, and member or subset relations are introduced on the basis of the associated quantifier specification. Our method for building explicit logical forms challenges Stallard's distinction of predicative and referential readings of metonymic expressions: it produces scopings that reflect proper quantifier dominance relations rather than pronominal accessibility conditions, and it allows for additional cases of pronominal reference. In addition, our method enables a more precise attachment of contextual specifications to related entities, and it supports reference resolution to metonymically related entities.

\section{References}

(Fass, 1991) Dan Fass. met*: A Method for Discriminating Metonymy and Metaphor by Computer. Computational Linguistics 17(1), pp. 49-90,1991.

(Fliegner, 1988) Michael Fliegner. HOKUSKOPUS Verwendung terminologischen Wissens bei der Analyse von Quantorenskopus und Distributivität. In Proc. of GWAI-88, pp. 112-117, 1988.

(Grosz et al. 1988) Barbara Grosz, Doug Appelt, Paul Martin, and Fernando Pereira. TEAM: An Experiment in the Design of Transportable Natural-Language Interfaces. Artificial Intelligence 32, pp. 173 243, 1987.

(Hobbs et al. 1993) Jerry Hobbs, Mark Stickel, Doug Appelt, and Paul Martin. Interpretation as Abduction. Artificial Intelligence, pp. 69-142, 1993.

(Horacek 1994) Helmut Horacek. Some Issues in Dealing with Metonymy. In Proc. of KONVENS-94, pp. 171-180, Vienna, Austria, 1994.

(Horacek 1996) Helmut Horacek. On Expressing Metonymic Relations in Multiple Languages. Machine Translation 11, pp. 109-158, 1996.

(Kayser 1988) Daniel Kayser. What Kind of Thing is a Concept. Computational Intelligence 4(2), pp. 158$165,1988$.

(Lakoff and Johnson 1980) George Lakoff and M. Johnson. Metaphors We Live By. Univ. of Chicago Press, 1980.

(Markert and Hahn 1997) Katja Markert and Udo Hahn. On the Interaction of Metonymies and Anaphora. In Proc. of IJCAI-97, pp. 1010-1015, Nagoya, Japan, 1997.

(Nunberg 1995) Geoffrey Nunberg. Transfers of Meaning. Journal of Semantics 12, pp. 109-132, Oxford University Press, 1995.

(Pustejovsky 1991) James Pustejovsky. The Generative Lexicon. Computational Linguistics 17(4), pp. 409441, 1991.

(Pustejovsky and Bouillon 1995) James Pustejovsky, and P. Bouillon. Aspectual Coercion and Logical Polysemy. Journal of Semantics 12, pp. 133-162, Oxford University Press, 1995.

(Sowa 1992) John Sowa. Logical Structures in the Lexicon. In J. Pustejovsky, S. Bergler (eds.): Lexical Semantics and Knowledge Representation, pp. 3960, Springer, 1992.

(Stallard 1993) David Stallard. Two Kinds of Metonymy. In Proc. of $A C L-93$, pp. 87-94, Columbus, Ohio, USA, 1993. 